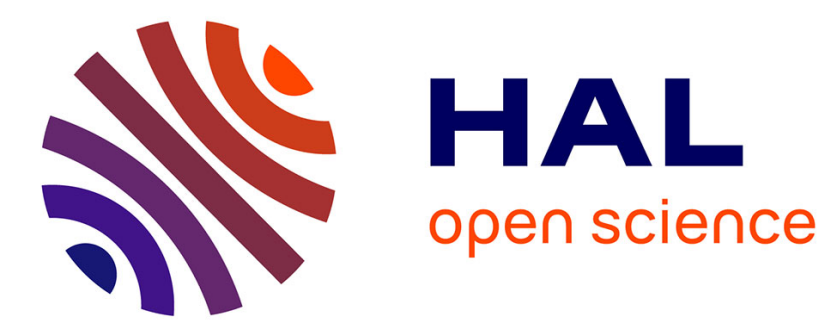

\title{
Pérou : traumas et violence d'État dans le roman Grandes Miradas de Alonso Cueto
}

Pierre Lopez

\section{To cite this version:}

Pierre Lopez. Pérou : traumas et violence d'État dans le roman Grandes Miradas de Alonso Cueto.

Cahiers d'Etudes Romanes, 2020, 10.4000/amerika.1419 . hal-03223828

\section{HAL Id: hal-03223828 \\ https://hal-amu.archives-ouvertes.fr/hal-03223828}

Submitted on 11 May 2021

HAL is a multi-disciplinary open access archive for the deposit and dissemination of scientific research documents, whether they are published or not. The documents may come from teaching and research institutions in France or abroad, or from public or private research centers.
L'archive ouverte pluridisciplinaire HAL, est destinée au dépôt et à la diffusion de documents scientifiques de niveau recherche, publiés ou non, émanant des établissements d'enseignement et de recherche français ou étrangers, des laboratoires publics ou privés. 


\title{
Pérou : traumas et violence d'État dans le roman Grandes Miradas de Alonso Cueto
}

\author{
Pierre Lopez \\ Aix Marseille Université, CAER, Aix-en-Provence, France
}

Résumé : Publié au Pérou en 2003, trois ans après la chute du président Alberto Fujimori et de son bras droit Vladimiro Montesinos, Grandes miradas était alors considéré comme le roman le plus abouti de Alonso Cueto. L'œuvre est basée sur une histoire vraie parue dans la presse péruvienne : l'exécution dans des conditions étranges et morbides du juge César Díaz Gutiérrez. L'auteur va réinvestir les faits en proposant dans son roman l'histoire d'une vengeance : celle d'une femme qui décide de tuer les assassins de son compagnon, un juge incorruptible. Elle se trouve confrontée à une violence jusqu'alors insoupçonnée, ou qu'elle avait toujours ignorée, mais qui va directement la faire pénétrer dans les arcanes de l'appareil d'État, au plus près de la gouvernance bicéphale du Pérou de la fin des années 1990. Parti d'un fait divers, l'auteur va ainsi déplacer l'action et lui donner une dimension autre, centrée sur la corruption, sur les pratiques d'intimidation, de chantage et de répression orchestrées par le bras droit du président Fujimori durant les derniers mois de sa gouvernance. En décrivant la violence d'État dans son expression la plus crue, l'auteur propose ainsi une critique acerbe, sans concession, sur les dix années de présidence de Fujimori.

Mots-clés : Roman, Alonso Cueto, Grandes miradas, violence d’État, trauma, Montesinos, Fujimori, Pérou.

Resumen: Publicada en Perú en 2003, tres años después de la caída del presidente Alberto Fujimori y de su mano derecha Vladimiro Montesinos, Grandes miradas fue considerada entonces como la novela más lograda de Alonso Cueto. La obra está basada en un hecho real que apareció en la prensa peruana: la ejecución en condiciones extrañas y morbosas del juez César Díaz Gutiérrez. El autor reinvertirá los hechos proponiendo en su novela la historia de una venganza: la de una mujer que decide matar a los asesinos de su compañero, un juez incorruptible. Se enfrenta a una violencia que nunca había sospechado o que había ignorado hasta entonces, pero que la llevará directamente a relacionarse con los turbios y sucios procedimientos del aparato estatal, lo más cerca posible del gobierno bicéfalo del Perú a finales de los 90. A partir de un suceso, el autor transformará la acción y le dará otra dimensión, centrada en las prácticas de corrupción, intimidación, chantaje y represión orquestadas por la mano derecha del presidente Fujimori durante los últimos meses de su gobierno. Al describir la violencia del Estado en su expresión más cruda, el autor ofrece una crítica acérrima y aguda de los diez años de Fujimori como presidente.

Palabras claves: Novela, Alonso Cueto, Grandes miradas, violencia de Estado, trauma, Montesinos, Fujimori, Perú. 
Publié au Pérou en 2003, trois ans après la chute du président Alberto Fujimori et de son bras droit Vladimiro Montesinos, le roman Grandes miradas ${ }^{1}$ était alors considéré comme le roman le plus abouti de Alonso Cueto ${ }^{2}$. L'auteur s'affirmait déjà comme l'un des écrivains les plus prometteurs et prolixes du paysage narratif péruvien durant une période que la critique avait appelée le «postboom ${ }^{3} »$. Cette appréciation s'est confirmée par la suite au-delà de cette période par la publication de plusieurs romans comme La hora azul (2005), Cuerpos secretos (2012), La pasajera ${ }^{4}$ (2015). Le point d'ancrage entre toutes ces œuvres s'articule autour d'un même noyau dynamique qui est nourri de cruauté, de violence, de violence envers les femmes, d'érotisme, de vengeance et de résilience. Un tel noyau permet à l'auteur de travailler l'élaboration et l'évolution de ses personnages sur une trame narrative toujours enracinée dans un contexte socio-économique et politique bien marqué, générateur de violence et de traumas. Alonso Cueto envisage ainsi de creuser l'âme humaine de ses concitoyens, celle des femmes victimes, mais aussi celle de l'être humain en général, cherchant à mettre en lumière, à la vue de «tous», les zones les plus sombres et insoupçonnées de notre tréfonds, de notre noirceur d'âme, mais dans un contexte extrêmement identifiable pour ses lecteurs péruviens.

De manière générale, l'argumentaire de ces trois narrations précédemment citées, prend racine dans un terreau historique et contextuel précis, qui se déploie en surface dans le temps, des années 1980 jusqu'au début du $\mathrm{XXI}_{\mathrm{e}}$ siècle, et en profondeur dans les traumas que les événements politiques ont générés, principalement à partir de l'émergence du Sentier Lumineux, de l'ère Fujimori et de son abrupte fin. Autrement dit, l'auteur construit ses personnages, principalement péruviens, en les marquant du sceau d'un trauma passé ou présent qui se révèle au fil de la narration. Alonso Cueto intègre ainsi dans ce contexte péruvien des éléments «d'actualité » parfois très précis dont certains ont été présentés par des médias comme des faits divers. C'est effectivement le cas pour le roman qui fait l'objet de notre analyse ainsi que pour La hora azul, qui sera publié deux ans après Grandes miradas et pour lequel Cueto recevra le Premio Herraldes en 2005. La hora azul est basé sur une histoire vraie parue dans la presse péruvienne : celle d'une jeune femme séquestrée sans raison par des militaires qui luttaient contre le Sentier Lumineux, et qui réussit à s'échapper. Contrairement à beaucoup d'autres femmes de la région d'Ayacucho, kidnappées, séquestrées clandestinement, utilisées comme objets sexuels puis exécutées d'une balle dans la tête, cette miraculée put ainsi être sauvée et permit de révéler certaines atrocités perpétrées par l'Armée sous couvert de lutte contre le terrorisme ${ }^{5}$.

Dans Grandes miradas, l'auteur s'appuie également sur un fait réel pour construire l'amorce de toute la trame. En juillet 2000, il prend connaissance dans le journal Caretas de la torture et de l'exécution dans des conditions étranges et morbides du juge César Díaz Gutiérrez. En complément d'information sur cette étrange «affaire », certains médias laissent sous-entendre que la mort de ce juge aurait un lien avec sa vie privée, plus précisément avec des mœurs homosexuelles des plus débridées. Si nous reprenons le document d'origine, le journal Caretas précise bien, pour défendre la probité et l'intégrité de la victime, que «Sobre sus preferencias sexuales se han tendido una serie de especulaciones, a cada cual más

\footnotetext{
${ }^{1}$ Alonso Cueto, Grandes miradas, Barcelona, Editorial Anagrama, 2003.

${ }^{2}$ Voir Joaquín Marco, «Grandes miradas de Alonso Cueto », 3 de febrero 2005, El Cultural, [s.p.] en ligne : <https://elcultural.com/Grandes-miradas> (consulté le 26 juin 2019).

${ }^{3}$ Sur cette période, voir par exemple José Luis de la Fuente, La nueva narrativa hispanoamericana: entre la realidad y las formas de la apariencia, Valladolid, Universidad de Valladolid, 2005.

${ }^{4}$ Alonso Cueto, La hora azul, Barcelona, Anagrama, 2005 ; Cuerpos secretos, Barcelona, Planeta, 2012 ; La Pasajera, Editorial Planeta Perú / Seix Barral, 2015.

${ }^{5}$ Voir Claire Sourp, «La hora azul en pos de la memoria traumática del Perú », Amerika, Amerika, 18 avril 2011, [s.p.], en ligne : <https://doi.org/10.4000/amerika.1419> (consulté le 16 juin 2019).
} 
escandalosas $^{6} \gg$. Mais, comme insiste le chroniqueur en reprenant les termes des personnes «proches » du juge : «Quienes lo conocieron dicen que aun si se descubriera que su crimen tuvo que ver con sus opciones sexuales, eso no borraría la limpieza de sus actos ${ }^{7}$ ». À partir de ces quelques "avis rapportés », lâchés de manière anodine au détour d'une phrase, dans une présentation générale vantant, malgré tout, les mérites de ce représentant de la justice et de la loi, la victime s'avère en fait «entachée » par ses sous-entendues «pratiques déviantes ». De ce fait, la gravité de l'acte dans l'assassinat barbare d'un juge reconnu exemplaire tend à être minimisée, voire reléguée à un vulgaire règlement de compte passionnel qui relèverait du domaine du privé et non du public.

Le lecteur Alonso Cueto, tout en reconnaissant que «La realidad siempre es más brutal que cualquier cosa que uno pueda escribir ${ }^{8} »$, va réinvestir les faits dans une fiction en les mettant directement en relation avec les arcanes de l'appareil d'État, au plus près de la gouvernance bicéphale du Pérou de la fin des années 1990. Fujimori et son entourage proche ainsi que Montesinos vont occuper leurs fonctions respectives dans la narration et constituent des personnages clés. Parti d'un fait divers, l'auteur va déplacer l'action et lui donner une dimension autre, centrée sur la corruption, plus en accord avec les pratiques d'intimidation, de chantage et de répression orchestrées par le bras droit du président Fujimori durant les derniers mois de sa gouvernance.

Dans un premier temps, le début du roman se déploie sur la description de Guido Pazos, juge intègre et incorruptible qui refuse, malgré les tentatives de corruption puis les menaces de mort, d'abandonner les poursuites pour détention d'arme qu'il maintient à l'encontre d'une connaissance de Montesinos. Ce dernier le fait alors sauvagement torturer et assassiner par ses sbires. Gaby, la fiancée de Guido, s'impose alors dans l'espace narratif. Cette jeune enseignante, issue de la bonne société de Lima, intègre la nouvelle de l'assassinat comme la fin d'un monde d'insouciance, d'un «âge d'or ». Ce personnage représente une partie de la population péruvienne qui se sentait en quelque sorte étrangère aux événements politiques, au terrorisme du Sentier Lumineux et surtout à celui exercé par l'État. Gaby se réfugiait dans un monde «confortable » et conforté par les valeurs d'une classe moyenne supérieure et, face à la perte de son compagnon, elle sombre dans l'incompréhension et le désarroi. Traumatisée par la mort du juge, elle sort de son apathie émotionnelle lorsqu'elle lit dans une revue à scandale un article diffamatoire sur la mort de son compagnon. Dans ce dernier, les préférences sexuelles de la victime sont mises en doute et l'exécution barbare est associée à un règlement de compte entre homosexuels. Les stratégies du contournement, du doute, du non-dit, du scandale pour cacher un autre «véritable » scandale, autrement dit, celui imputé au terrorisme d'État, se révèlent à Gaby. Elle décide alors de se venger et de tuer Montesinos et ses sbires. Pour mener à bien son projet, elle décide de changer de vie : elle abandonne son poste d'enseignante et s'inscrit dans une école de secrétariat dont la directrice est proche du gouvernement et plus précisément de Montesinos. En effet, cette responsable d'un établissement d'enseignement pour jeunes femmes, personnage autoritaire, fourbe et ambitieux qui a pignon sur rue et qui côtoie la bonne société de Lima, fournit les filles lors des orgies organisées par le bras droit du président. Montesinos affectionne particulièrement ces parties fines pour son plaisir personnel mais également, et surtout, pour récompenser ses plus proches collaborateurs ou ceux qui n'auront d'autre choix que de le devenir, étant donné

\footnotetext{
${ }^{6}$ Ramiro Escobar la Cruz, «El Magistrado Asesinado », Caretas, 20 de julio 2000, [s.p.], en ligne : <http://web.archive.org/web/20010304090937/http://www.caretas.com.pe/2000/1628/articulos/magistrados.phtm 1> (consulté le 26 juin 2019).

${ }^{7}$ Ibid., [s.p.].

${ }^{8}$ Rosa Mora, «Alonso Cueto traza en "Grandes miradas" un retrato estremecedor del Perú de Fujimori », El País, 27 de marzo 2005, [s.p.], en ligne : <https://elpais.com/diario/2005/03/27/cultura/1111878004_850215.html> (consulté le 26 juin 2019).
} 
que tous ces moments «d'intimité partagée » sont discrètement filmés. Au cours de la narration, certains ébats sont intérieurement commentés par Montesinos et révèlent chez ce personnage un besoin obsessionnel de dominer l'autre. Ce désir de domination, de contrôle, s'exerce par le regard, un regard libidineux, intrusif, pénétrant; les faits sont ainsi "grandement regardés », pour renouer avec le titre du roman, que nous pouvons traduire littéralement par «les grands regards ». Gaby va également apprendre à « regarder»; elle va découvrir un monde nouveau, un monde qu'elle côtoyait sans s'en apercevoir et c'est en partie sa nouvelle complice, la rédactrice de l'article diffamatoire sur Guido, qui, empreinte de remords, lui ouvrira les yeux. Gaby finit par remonter la piste des assassins de son compagnon. Elle pénètre ainsi dans les arcanes de tout un système oppressif, barbare, mais qui est protégé par une forme de déni et par l'imposition d'un discours officiel. Ce système est alimenté et maintenu par une violence extrême dans laquelle la jeune enseignante, qui était pourtant promise à un avenir doré, se fourvoie pour se transformer en une Diane chasseresse impitoyable et sanguinaire. Elle tue l'un des assassins de Guido, l'un des sbires qui est présenté comme un tortionnaire sadique, appliqué dans son ouvrage décrit comme un acte clinique. La torture est présentée comme une pratique ritualisée, «imposée » par Montesinos. En tant que chef des services secrets, mu par son délire pathologique de vouloir tout voir, il ne manquait pas de faire filmer les scènes de torture avant que le tortionnaire ne passe à l'exécution. Dans le roman, l'exécution de Guido ne déroge pas à la règle. Gaby découvre par étapes toutes ces pratiques. Dans sa quête sanguinaire, elle réussit même, en séduisant la directrice de l'école de secrétariat, à avoir une entrevue avec Montesinos dans une chambre d'hôtel. Mais au moment de passer à l'acte et de lui trancher la gorge, elle flanche et se fige. Aussitôt arrêtée, torturée par les hommes de Montesinos, elle est laissée pour morte dans l'une des geôles clandestines des services secrets, mais les amis de Gaby arrivent malgré tout à la faire sortir de sa cellule, au moment où éclate le scandale de la vidéo de Montesinos. La descente aux enfers de Gaby s'achève avec la fin du règne de Montesinos, provoquée par une vidéo qui a été récupérée par un journaliste et dans laquelle on voit (disons plutôt que tout le pays a vu) comment le bras droit de Fujimori donne une liasse de billets pour corrompre un chef politique.

Ainsi, comme nous venons de l'évoquer dans ce bref résumé de l'œuvre, au cours de son périple Gaby croise une série de personnages représentant différents secteurs de la société. Les mass médias sont bien représentés. Ángela est un personnage important dans la mesure où elle est la rédactrice d'un journal à scandale proche du gouvernement dont le but est de salir tous ceux qui s'opposent au président et à Montesinos. Elle deviendra, prise de remords pour avoir souillé l'image du juge Guido, l'amie et la complice de Gaby et, d'une certaine manière, elle incarnera un espoir dans un monde où règne la corruption. Le monde des médias est également représenté par le personnage de Javier, un journaliste vedette, présentateur de JT également à la solde du gouvernement. L'auteur se sert ainsi de ces deux personnages pour démanteler l'une des armes de fond de l'ère Fujimori qu'est la presse. Et dans ces deux cas, nous avons deux modalités d'une presse infiltrée par le gouvernement. L'une est considérée comme proche du peuple, la «prensa chicha », presse à scandale bon marché qui, à travers les gros titres à sensation et certains cas emblématiques, manipule ses lecteurs à coups de sentences moralisatrices directes ou sous-entendues, notamment envers les opposants au gouvernement. L'autre presse est plus « officielle », celle des JT et des stars de l'information des petits écrans, celle qui se targue de neutralité en brandissant une éthique professionnelle. Cela dit, grâce à la diffusion d'une vidéo montrant un député de l'opposition se laissant corrompre en acceptant une liasse de billets, c'est ce même pouvoir de la presse qui contribuera à la chute du gouvernement Fujimori.

Le monde de la justice, avec ses failles, est également présent avec le personnage de Artemio, un secrétaire et ami du juge qui se laissera corrompre en aidant les sbires de Montesinos à 
rentrer dans l'appartement de Guido, devenant ainsi complice de son exécution. Parmi ces sbires se trouve le frère de Ángela, issu des quartiers défavorisés. Ce personnage dont les parents ont été exécutés par le Sentier Lumineux, de victime deviendra bourreau et sera « fraîchement » initié aux techniques de nettoyage des opposants au régime au cours de rites barbares et sacrificiels réalisés au nom de l'ordre social et politique. Tous ces personnages, malgré le déni ou l'acceptation de la violence d'État, finiront par aider Gaby, lui évitant la mort au cours d'une sorte de rédemption ou résilience qui s'achève par la libération de la victime sacrificielle.

Le roman propose ainsi une recréation de la violence politique dans tous ses soubassements avec comme fil conducteur la vengeance du personnage principal. L'œuvre est en fait un roman choral, où les pensées parfois les plus intimes sont révélées, celle de Gaby mais également celles de Montesinos et de Fujimori.

Vladimiro Montesinos, qui était surnommé le «Raspoutine des Andes », est décrit comme le grand organisateur, la pièce maîtresse d'un régime gangréné par la corruption à tous les niveaux de la société, des secteurs les plus pauvres jusqu'aux plus grands représentants du gouvernement, sans oublier les plus hauts dignitaires de l'Armée. L'auteur fait de lui un personnage sadique, satanique, pervers, paranoïaque et bien entendu voyeur, « el ángel de una Anunciación maligna ${ }^{9} »$. Cueto travaille en profondeur son personnage par le jeu des narrations à la troisième personne proposées par un narrateur omniscient ; des narrations qui alternent avec des monologues intérieurs et des descriptions faites par le personnage de Gaby : «cráneo húmedo, las mejillas altas, los ojos secos de ofidio, la nariz afilada, la piel de escamas y puntos, el grosor de la sonrisa ${ }^{10}$ ». Par ce jeu de narrations qui intègre des descriptions physiques mais aussi morales, l'auteur donne une épaisseur psychologique au personnage ce qui renforce l'horreur et la répugnance qui se dégagent de lui, et à travers lui, de tout le gouvernement. Montesinos se construit comme étant «una revelación del subsuelo $^{11}$ », un être infâme, génie du mal dont les perversions, sorte de vadémécum funeste, imprègnent tout l'appareil gouvernemental. L'auteur intègre aussi les descriptions en sa faveur, reproduisant ainsi le discours officiel pour justifier la politique répressive du gouvernement, transformant le monstre en Ange rédempteur. Don Ramiro, le supérieur hiérarchique du juge Guido, voit en Montesinos «Un enviado de Dios ${ }^{12}$ »; un adoubement qui fait de l'institution judiciaire un complice de choix dans le système répressif. Cueto considère que «El poder extraordinario de Montesinos se basaba en tres elementos a través de los cuales controlaba todos los estamentos de la sociedad: la comunicación, la administración judicial y la militar ${ }^{13} \gg$. La presse favorable au gouvernement est aussi représentée par Don Osmán, l'un des dirigeants de la presse officielle, dont le journal est directement sous le contrôle de Montesinos. Le personnage décrit ce dernier comme suit :

Bueno, es un señor maravilloso. Un enviado del Señor en verdad, te digo. Un hombre que trabaja veinte horas diarias. Un enviado de Dios, no sé dónde estaríamos sin él. Mira cómo está Colombia con los guerrilleros metidos y en cambio nosotros aquí comiendo tan tranquilitos, pues ${ }^{14}$.

Mais c'est dans la perversion que le personnage de Montesinos acquiert le plus de profondeur et Cueto a su exploiter sans concession le fameux vice du dirigeant : ses vidéos. Montesinos avait une obsession qui consistait à tout faire enregistrer, les tortures comme les scènes de

\footnotetext{
${ }^{9}$ Alonso Cueto, Grandes miradas, op. cit., p. 116.

${ }^{10}$ Ibid., p. 15.

${ }^{11}$ Ibid., p. 264.

12 Ibid., p. 73.

${ }^{13}$ Rosa Mora, «Alonso Cueto traza en "Grandes miradas" un retrato estremecedor del Perú de Fujimori », art. cit., [s.p.].

${ }^{14}$ Alonso Cueto, Grandes miradas, op. cit., p. 214.
} 
corruption, ce qui le conduira à sa perte. Ce sont les fameuses «vladivídeos ${ }^{15}$ » qui seront commentées par Luis Jochamowitz dans son œuvre Vladimiro. Vida y tiempo de un corruptor $^{16}$ et à qui Alonso Cueto rend hommage dans un petit texte proposé en guise de préface ou d'avertissement : «En concordancia con su naturaleza novelesca, los hechos que se narran en este libro pertenecen al ámbito de la ficción. En algunos casos, están inspirados en pasajes de la magnífica biografía Vladimiro. Vida y tiempo de un corruptor, de Luis Jochamowitz ».

L'auteur a regardé beaucoup de «Vladivídeos », mais il précise qu'il s'était refusé à visionner celles qui portaient sur les tortures. Les vidéos sont révélatrices pour l'auteur du regard que portait Montesinos sur le genre humain :

Él daba por sentado la naturaleza corruptible y clandestina de las personas, contaba con ella. Instauró un gran sistema de vigilancia en el que utilizaba a todo el mundo, chóferes, empleadas domésticas, tenía cámaras que lo grababan todo. La suya era una enorme mirada sobre la sociedad ${ }^{17}$.

Ainsi, si ces vidéos révèlent tout un système de corruption qui touche tous les secteurs de la société, elles sont également révélatrices de la perversité de l'individu. Montesinos manifestait une certaine jouissance lorsqu'il regardait ses vidéos. Dans le roman, outre le plaisir qu'il ressent comme affirmation de son pouvoir, de son contrôle sur la société, c'est une véritable jouissance sexuelle qui s'empare de lui en regardant des scènes de viols, de torture (qu'il appelait « orgías médicas $\left.{ }^{18} »\right)$, de corruption. Mais il va au-delà aussi de cette dimension sexuelle, car les vidéos provoquent en lui un délire de grandeur empreint de mysticisme :

Montesinos enciende la pantalla, se mira entregando un fajo de billetes, se concentra en las cesiones en la casa del congresista que acepta el dinero.

El cuarto oscuro apenas se ilumina con la pantalla. La televisión es el sol de ese universo negro. Él es el centro de la televisión. Estira las piernas. La oscuridad del cuarto hace más ancha y profunda la mirada. La oscuridad es su hogar. Desde ese agujero puede ver pasar presidentes y ministros y asesores, todos reducidos por el fulgor de la vida pública. La grandeza de la oscuridad es suya. La luz descubre y vulnera, empequeñece los cuerpos. Él sabe, Vladi, que la verdadera vida es el secreto. Dios existe porque nadie lo ve. El que puede ver y no ser visto. Eso soy. No un hombre. Una fuerza, un rayo oscuro, permanente. Un ángel de humo blanco se confunde con la neblina ${ }^{19}$.

Montesinos s'octroie une dimension semi-divine, il est celui qui contrôle tout sans être vu, celui qui a un ascendant sur les corps, tous les corps de ses amis et ennemis, avec également la prétention de voir à l'intérieur des hommes : «Aprisionar la última región de los cuerpos, la matriz de pestilencia de las almas, el botín ajeno: tocar las heces, el pubis, el pene, la lágrima cerrada, con los ojos [...] tomar el poder en los cuerpos ${ }^{20} \gg$.

Fujimori quant à lui est également décrit comme un être paranoïaque, complexe et complexé par ses origines modestes qu'il ressent comme un obstacle à son intégration parmi l'oligarchie péruvienne. L'auteur pointe principalement le mensonge et le vol comme des «valeurs» endémiques de sa nature et de sa propre corporéité :

La costumbre de mentir, de robar, de hacer matar, el gran estrecho palacio de su vida. ¿Desde cuándo? Siempre, siempre, siempre. Él se alimenta, respira de la mentira, el robo, la muerte, los órganos de su cuerpo. El corazón del sistema circulatorio y digestivo de estos órganos es el hábito, un músculo metódico de intentos de engañar al mundo. Engañar y robar y respirar, ordenar la realidad, hacer y disponer y aplanar la superficie, la realidad puede ser tan simple, él puede regirla todas las madrugadas

\footnotetext{
${ }^{15}$ Vidéos n。 872 et 873 «Reunión Alex Kouri-DR, 28 de enero de 1998 »; «Vladivídeo 02, Alex Kouri y Vladimiro Montesinos » en ligne : <https://www.youtube.com/watch?v=SVibUHs8UwM> (consulté le 26 juin 2019).

${ }^{16}$ Luis Jochamowitz, Vladimiro. Vida y tiempo de un corruptor, Lima, Ediciones El Comercio, 2002.

${ }^{17}$ Rosa Mora, «Alonso Cueto traza en "Grandes miradas" un retrato estremecedor del Perú de Fujimori », art. cit., [s.p.].

${ }^{18}$ Alonso Cueto, Grandes miradas, op. cit., p. 41.

${ }^{19}$ Ibid., p. 257.

${ }^{20}$ Ibid., p. 187.
} 
desde su cuarto. Las cifras de las cuentas, los rápidos cadáveres, los muertos sin nombre. Un presidente condecorado de billetes y de sangre ${ }^{21}$.

Ces descriptions sans concession du président, sous-tendues par un jeu de questions / réponses (dans notre exemple, «¿Desde cuándo? ») s'organisent comme une autopsie opérée en guise de réponse mettant ainsi à nu la dynamique interne d'un corps et d'un esprit mus par le mensonge, le lucre et la mort. Le rythme donné par des figures rhétoriques accumulatives telles que la concaténation réalisée à partir des membres du corps (cuerpo / sistema circulatorio / digestivo / músculo / mundo) maintient une sorte d'halètement compulsif qui accompagne la propagation de cette dynamique interne vers le monde extérieur («engañar al mundo / ordenar la realidad»). Ainsi, dans cette description presque clinique du personnage de Fujimori proposée «au regard de tous » comme une trépanation de son esprit, de son tréfonds, de son âme, la séparation entre le personnage et la personne qui a gouverné le pays durant une décennie devient aussi mince que la lame d'un scalpel. L'auteur n'omet surtout pas le lucre à la galerie des vertus de Fujimori, il en fait l'un des «membres vitaux » de son organisme, détruisant ainsi l'image que s'était donnée le président ; celle que ce dernier avait mis en avant lors de sa première campagne présidentielle : un homme politique près du peuple, loin de l'oligarchie et initiateur d'une ère nouvelle de paix et de prospérité. Cela dit, ce personnage n'a pas le monopole dans l'espace narratif, l'œuvre est très centrée sur Montesinos et sur ses liens avec le président ; des liens à la fois si particuliers et si révélateurs d'un système politique et social national que commente le narrateur :

Fujimori pertenecía a la clase baja, y, Montesinos, a la adinerada. Fujimori tuvo una vida dura de niño. Consiguió el poder económico, y luego, el político, pero nunca fue aceptado por la clase alta de Lima. Era hermético y extraño, y acabó entregándose a Montesinos, al que miraba como un oráculo. [...] El presidente Fujimori. Ahora se llama así. ¿Quién sería después? La muralla de su presente lo había atrincherado. Su personalidad, un laberinto lleno de antesalas y exámenes de ingreso. Nadie iba a entrar allí, nadie iba a saber quién era. Montesinos cree que lo conoce. No lo conoce, lo domina, Montesinos es un órgano de su cuerpo. Para huir de Montesinos, viajaba a los pueblos de la sierra, a inauguraciones, ceremonias de donación, fiestas patronales ${ }^{22}$.

L'auteur a su travailler en profondeur ses personnages à partir d'éléments contextuels qui relèvent de la politique en général, de la gouvernance et de la personnalité de ses acteurs comme dans ce dernier exemple où il est fait allusion à l'hermétisme du président Fujimori. Par un travail de «fictionnalisation» à la fois esthétique et prospectif qui, dans la construction des «mondes intérieurs » de ses personnages, emprunte les cheminements de la psychanalyse, Cueto construit un véritable monde qui était perçu à la fois si près et si loin par ses contemporains. Par le truchement de la fiction, il établit un rapprochement avec la sphère secrète et privée de la gouvernance dont la communication et l'image étaient entièrement sous contrôle de Montesinos, lequel maintenait une distance «sécuritaire » que personne n'osait réduire lorsque cela relevait du domaine du privé. En revanche, les mass médias diffusaient l'image d'un président soucieux envers les plus démunis de la Sierra, lui-même issu du « peuple », garant malgré l'inflation et une démocratie fragile d'un ordre économique et d'une stabilité politique. Dans le domaine politique, Fujimori est celui qui a su faire preuve de fermeté, d'autoritarisme, en provoquant par la force un «autogolpe », mais c'est surtout celui qui, deux ans après sa première élection, a pu revêtir l'image du sauveur de la nation : celui qui, avec le chef des services secrets et à travers lui des forces anti-terroristes, a mis fin au règne du Sentier Lumineux, avec la capture de son chef Abimael Guzmán. Rappelons comment cette capture a été mise en scène et largement diffusée : Abimael Guzmán est « exposé » en train d'invectiver ses observateurs, habillé en prisonnier dans une cellule ${ }^{23}$. Nul

\footnotetext{
${ }^{21}$ Ibid., p. 88.

${ }^{22}$ Ibid., p. 137.

23 Voir par exemple «La captura de Abimael Guzmán, líder de Sendero Luminoso, narrada por los protagonistas »[vídeo], en ligne : 〈https://www.youtube.com/watch?v=2htQq1yjFiw> (consulté le 26 juin 2019).
} 
doute que ces images ont contribué à affaiblir l'aura semi-mythique de l'homme le plus recherché du Pérou, à exorciser ce qui pour beaucoup représentait l'origine du mal, mais elles ont aussi permis d'asseoir, $d$ ' « officialiser » une politique anti-terroriste très répressive faisant régner un terrorisme d'État, notamment dans certaines régions de la Sierra. À travers son roman, l'auteur se propose justement de revenir sur certaines images, sur certaines personnalités de l'ère Fujimori et sur tout un contre-discours ou résistance à un système qui ont été minimisés, occultés, anéantis au nom d'une politique sécuritaire qui a fait des milliers de victimes.

Comme nous l'avons déjà souligné, l'auteur part d'un fait divers pour construire son récit, l'exécution du juge Gutiérrez. Mais Cueto s'intéressera à l'affaire, il entrera en contact avec la famille du juge de qui il dira «Era muy modesta, decente y bondadosa ${ }^{24}$ ». Il visitera sa maison, son bureau au Palais de justice et fréquentera ses proches. Ce juge va, d'une certaine manière, « reprendre vie » dans le roman sous les traits du juge Guido Pazos, juge intègre et incorruptible, soumis à des pressions par sa hiérarchie et victime de menaces qui aboutiront à son exécution. Le narrateur le décrit comme «un caballero aterrizado en el Palacio de Justicia, un sacerdote sin cáliz, un santo sin aureola ${ }^{25} »$ et son bureau se transforme en un autel d'où il prodiguait la justice : «el altar en el que Guido oficiaba la misa de su probidad todos los días ${ }^{26}{ }$. Cueto s'est par conséquent largement inspiré de la vie et de la formation du véritable personnage du juge Gutiérrez pour construire son personnage de Guido. Mais il va au-delà en lui conférant dans la narration un caractère hagiographique empreint d'un esprit messianique dans sa lutte contre le mal. Il fait de lui l'unique représentant des valeurs et d'un monde perdus, ou idéalisés, où la justice était, ou est, garante du respect des droits et de la démocratie. Il confère ainsi un caractère sacré à la fonction de juge : «el juzgado no es una oficina, un juez no es un trabajador así nomás, es un dios de los hechos, les da su valor, los hace significar algo, un juez es una brújula, alguien a quien los justos del mundo observan con esperanza $^{27} \gg$. Le personnage de Guido fera même de l'autodérision sur le caractère messianique qu'il prête à sa fonction de juge :

imagínate que estoy estudiando a estas alturas, yo era seminarista en realidad, iba para cura pero me salí y aquí estoy, pues. Pasé de cura a abogado. Bueno, pero los curas y abogados en algo se parecen. Que siempre andan de negro y bien vestidos, ¿no ${ }^{28}$ ?

À partir de son exécution, c'est le personnage de Gaby qui prend le relais dans la narration et il sera présent, en creux, à travers la transformation de sa compagne.

Elle est, avec Montesinos, l'un des personnages les plus travaillés qui vivra l'assassinat de son fiancé comme un véritable trauma et qui dans sa soif de vengeance mènera une sorte de via crucis intérieure. L'auteur va faire de son personnage une victime, certes, mais surtout un réceptacle et un vecteur de violence : la violence engendre la violence. René Girard utilise la métaphore du feu pour illustrer cette dynamique :

Toujours, semble-t-il, le moment arrive où l'on ne peut plus s'opposer à la violence que par une autre violence; peu importe, alors, que l'on réussisse ou que l'on échoue, c'est toujours elle la gagnante. La violence a des effets mimétiques extraordinaires, tantôt directs et positifs, tantôt indirects et négatifs. Plus les hommes s'efforcent de la maitriser, plus ils lui fournissent des aliments ; elle transforme en moyens d'action les obstacles qu'on croit lui opposer ; elle ressemble à une flamme qui dévore tout ce qu'on peut jeter sur elle, dans l'intention de l'étouffer ${ }^{29}$.

C'est la confrontation avec cette violence, son intégration et sa gestion qui porte toute la tension dans le traitement du personnage de Gaby. Elle va se transformer en une autre Gaby,

\footnotetext{
${ }^{24}$ Rosa Mora, « Alonso Cueto traza en "Grandes miradas" un retrato estremecedor del Perú de Fujimori », art. cit., [s.p.].

${ }^{25}$ Alonso Cueto, Grandes miradas, op. cit., p. 23.

${ }^{26}$ Ibid., p. 23.

${ }^{27}$ Ibid., p. 26.

${ }^{28}$ Ibid., p. 25

${ }^{29}$ René Girard, La violence et le sacré, Paris, Hachette Littérature,1972, p. 51.
} 
aux antipodes de ce qu'elle était avant la mort de Guido ; celle qui ne voyait pas et se laissait porter par sa vie confortable, devra regarder, voir et se voir. Elle va explorer et exploiter toute la dimension maléfique qu'elle a en elle pour mener à bien sa vengeance : «Debía regresar a ese cuerpo. Buscar a través de él, en el comienzo de su infancia, el tesoro del mal que siempre había tapiado con sus maneras y razones ${ }^{30} »$. Dans la chambre de l'hôtel, au moment fatidique où elle s'apprête à trancher la gorge de Montesinos, alors qu'il est sur elle pour la posséder, il lui demande qui elle est. Elle lui répondra dans le creux de l'oreille, je suis toi : «Tú susurra-. Soy tú ${ }^{31} »$. Mais lorsqu'une «mystérieuse peine l'envahit et la fige », elle rompt avec son désir de vengeance, avec cette spirale de la violence qui la fait «être autre » et dans ce cas cet autre est la représentation du mal absolu. L'expérience du trauma, de la violence qu'elle intègre dans sa chair et qu'elle subira lors de sa torture ponctue toute l'évolution du personnage. Ce voyage intérieur constitue en soi une trame intérieure parallèle à l'élaboration par étapes de la vengeance et Cueto a su exploiter la notion même de trauma dans l'exploration qu'il fait du mal.

Derrière ce trauma, c'est aussi tout un travail de deuil qui ne se réalise pas, car il ne peut pas se faire pour le personnage tant que le terrorisme d'État est maintenu et que les valeurs et la confiance envers les institutions ne sont pas garanties. Ce n'est qu'à la fin du roman, avec la chute du monstre bicéphale, que Gaby semble s'apaiser. Comme le confirment certains psychanalystes, le deuil doit se faire dans une inscription collective :

Le travail psychique du deuil, qui aboutit à admettre la perte et la séparation de l'être aimé, à l'établir en soi dans l'ambivalence des sentiments, et à reprendre, à cette occasion, le travail du deuil des premiers objets d'amour, s'étaye toujours sur une inscription collective, sociale, culturelle ou religieuse ; il prend appui sur des actes rituels et sur des énoncés communs qui disent quelque chose d'important et de nécessaire sur l'origine, sur la fin et sur la succession des générations. En ce sens il n'y a pas de deuil strictement privé, quand bien même le travail du deuil est, comme tout travail psychique, une création qui engage la singularité intime de chaque sujet ${ }^{32}$.

De manière concomitante, dans l'exploration de la violence à travers la vengeance, l'auteur travaille sur le deuil, sur sa «singularité intime» mais également sur son inscription collective, sociale et politique. A la fin du roman, Gaby se réfugie dans la quiétude de sa chambre. Au préalable, lors de son entrevue avec le père de Guido qui l'apaise, elle fait de son deuil non une nécessité de pardonner mais de comprendre. Face à la violence, le besoin de comprendre s'impose; comprendre le choix de la résistance face à cette violence, réinvestissant ainsi dans l'exemplarité de Guido, dans sa mémoire, cette dimension hagiographique, afin de laisser la place à l'espoir :

-O sea, nadie lo va a decir nunca pero yo creo que Guido con lo que hizo, con lo que resistió, o sea con ese poquito que aguantó y gente como él, yo creo que ayudó a cambiar las cosas, o sea yo creo que quien sea, quien sea que resiste un poco, en cualquier sitio, o sea el que se niega a aceptar la mugre que alguien le impone, ese tipo es el que ha cambiado o está cambiando algo, o sea es el que nos salva un poco. [...] o sea a veces pienso que el hecho de que estemos vivos a lo mejor se lo debemos a alguna gente a la que no conocemos, o sea esa gente que hizo algo un día, algo como parar a un terrorista o no obedecer a un corrupto, y a lo mejor si nosotros estamos aquí es por esa gente que hizo algo una vez y ahora esa gente descansa sola, nadie se acuerda de ellos, ¿no ${ }^{33}$ ?

En décrivant la violence d'État dans son expression la plus crue, en décrivant les scènes de torture et le trauma qu'elle inflige par ricochets ou parce qu'il est vécu physiquement, l'auteur adopte une position face à l'expression de l'horreur. Cette position rejoint certains psychanalystes qui ont traité des patients victimes de la dictature en Argentine :

Mais un analyste sait aussi, en raison même de son métier, que l'acte de parole n'est pas innocent, et que convoquer l'effroi dans l'espace de parole n'est pas exempt de dangers. Comme par exemple celui de la fonction colonisatrice de la théorie... cette illusion que nous savons de quoi il s'agit. Ou bien, il y a le

\footnotetext{
${ }^{30}$ Alonso Cueto, Grandes miradas, op. cit., p. 183.

${ }^{31}$ Ibid., p. 265.

${ }^{32}$ Janine Puget et al., Violence d'état et psychanalyse, Paris, Dunod, 1989, p. 200-201.

${ }^{33}$ Alonso Cueto, Grandes miradas, op. cit., p. 299.
} 
risque de la capture, par la narration, dans la scène sadique, fascination du visuel-hallucinatoire. Entre les deux, nous ne disposons que d'un espace très réduit pour éviter que l'horreur ne vienne se superposer à l'acte de penser. Et pourtant c'est bien là, dans cette faille qui mène aux abîmes de l'horreur, qu'il est nécessaire de construire l'espace du penser, en se démarquant de l'hallucination et en créant un intervalle entre l'horreur et la réflexion la concernant. Il me semble -position qui sera attaquée par les révolutionnaires et les scientifiques- que c'est un lieu possible pour le psychanalyste que de tenter l'aventure de symboliser le patrimoine mortifère que nous a légué la décennie noire et, au travers de son élaboration, de le rendre transmissible. Il faut s'opposer à l'oubli et à la vengeance par l'inscription de la mémoire et sanctionner le crime par la construction d'une histoire ${ }^{34}$.

Cueto propose ainsi dans son roman une véritable radiographie de la société péruvienne à partir d'une vengeance qui va révéler les arcanes du pouvoir central. Son récit, considéré comme un «roman politique ${ }^{35}$ », fait de son auteur l'un des écrivains cronistas de l'horreur et de la violence qui ont marqué le pays entre 1980 et 2000 ; un pays dirigé par le tandem Fujimori-Montesinos, qui sous couvert d'une répression dure et affirmée contre le terrorisme, a su imposer un terrorisme d'État. Mais l'auteur contribue, par la fiction, à penser et à panser les traumas causés par cette violence d'État. Dans le dialogue qui termine le roman, dialogue entre Gaby et le père de Guido, elle préfère remplacer le verbe «perdonar » par « entender », et sauver de l'oubli, pour aller malgré tout de l'avant, ce qu'elle appelle une «memoria ejemplar».

\section{Bibliographie}

CUETO, Alonso, Grandes miradas, Barcelona, Editorial Anagrama, 2003.

CUETO, Alonso, La hora azul, Barcelona, Anagrama, 2005.

CUETO, Alonso, Cuerpos secretos, Barcelona, Planeta, 2012.

CUETO, Alonso, La Pasajera, Editorial Planeta Perú / Seix Barral, 2015.

DE LA FUENTE, José Luis, La nueva narrativa hispanoamericana: entre la realidad y las formas de la apariencia, Valladolid, Universidad de Valladolid, 2005.

ESCOBAR LA CRUZ, Ramiro, «El Magistrado Asesinado », Caretas, 20 de julio 2000, [s.p.], <http://web.archive.org/web/20010304090937/http://www.caretas.com.pe/2000/1628/articulo s/magistrados.phtml> (consulté le 26 juin 2019).

GIRARD, René, La violence et le sacré. Paris, Hachette Littérature, 1972.

GUICHOT, Elena, «Desvelando al Perú: La revelación de las Grandes Miradas », Altre Modernità: Rivista di studi letterari e culturali, agosto 2013, p. 159-172, $<$ https://www.google.com/url?sa=t\&rct=j\&q=\&esrc=s\&source=web\&cd=2\&cad=rja\&uact=8 \&ved=2ahUKEwjKgeOyqozjAhUHfxoKHTkNBSUQFjABegQIABAB\&url=https\%3A\%2F \%2Fwww.researchgate.net\%2Fpublication\%2F292158935_Desvelando_al_Peru_La_revelaci on_de_las_Grandes_Miradas\&usg=AOvVaw0pZkKM0OXUpFyZ3tX0j9xJ> (consulté le 26 juin 2019).

\footnotetext{
${ }^{34}$ Janine Puget et al., Violence d'état et psychanalyse, op. cit., p. 52.

${ }^{35}$ Joaquín Marco, «Grandes miradas de Alonso Cueto », art. cit., [s.p.].
} 
JOCHAMOWITZ, Luis, Vladimiro. Vida y tiempo de un corruptor, Lima, Ediciones El Comercio, 2002.

MARCO, Joaquín, «Grandes miradas de Alonso Cueto », El Cultural, 3 de febrero 2005, [s.p.], <https://elcultural.com/Grandes-miradas> (consulté le 26 juin 2019).

MORA, Rosa, «Alonso Cueto traza en "Grandes miradas" un retrato estremecedor del Perú de Fujimori », El País, 27 de marzo 2005, [s.p.], <https://elpais.com/diario/2005/03/27/cultura/1111878004_850215.html> (consulté le 26 juin 2019).

PUGET, Janine et al., Violence d'état et psychanalyse, Paris, Dunod, 1989.

SOURP, Claire, «La hora azul en pos de la memoria traumática del Perú », Amerika, 18 avril 2011, [s.p.], <https://doi.org/10.4000/amerika.1419> (consulté le 16 juin 2019). 